\title{
361 HETEROGENEITY OF PD-1HI T CELLS ASSOCIATES WITH RESPONSE TO PD-1 BLOCKADE IN HEPATOCELLULAR CARCINOMA
}

Assaf Magen*, Assaf Magen, Pauline Hamon, Myron Schwartz, Thomas Marron, Alice Kamphorst, Miriam Merad. MSSM, New York, NY, USA

Background Blockade of the PD-1 pathway is a therapeutic strategy to reinvigorate $\mathrm{T}$ cell responses against tumors, and when combined with other biologic therapies in the first line setting this achieves significant clinical response in about 25\% of hepatocellular carcinoma (HCC) patients. We hypothesize that phenotypic diversity of tumor infiltrating $\mathrm{T}$ cells can explain, at least partially, the disparate clinical responses to immunotherapy.

Methods Here, we analyze the molecular diversity of $\mathrm{T}$ cells in tumor, adjacent tissue and tumor-draining lymph node (dLN) by single-cell RNA sequencing of tissue from 23 patients with early stage HCC treated by neoadjuvant PD-1 blockade (NCT03916627).

Results We identify distinct subsets of PD-1hi T cells with varying degrees of exhaustion and effector gene programs. Compared to parallel analysis of untreated HCC tumors, we observed that PD-1 blockade resulted in expansion of PD-1hi $\mathrm{T}$ cells in the tumor, regardless of clinical response. PD-1hi $\mathrm{T}$ cells subsets were highly clonal and enriched in the tumor compared to adjacent tissue, suggesting specificity to tumor antigens. Remarkably, within the PD-1hi $\mathrm{T}$ cell population we find an association between specific transcriptomic phenotype that correlates with response to $\mathrm{PD}-1$ blockade. Using $\mathrm{T}$ cell receptor (TCR) sequencing to study the differentiation patterns between $\mathrm{T}$ cell states, we found that clonotypes present among expanded PD-1hi $\mathrm{T}$ cells were also found in CD8 effector cells; these data identify characteristic clonally related $\mathrm{T}$ cell populations that are enriched in clinical responders. Furthermore, we find that dLN harbor clonotypes of PD-1hi T cells expanded in tumor. In the dLN, these potentially tumor-specific $\mathrm{T}$ cells have features of activation and exhaustion suggesting a continuous role of $\mathrm{dLN}$ in anti-tumor responses. This study suggests a link between particular PD-1hi $\mathrm{T}$ cell subsets and responsiveness to PD-1 blockade.

Conclusions These results will be corroborated with 8 additional patient samples in which we will further analyze the role of tumor-specific $\mathrm{T}$ cells in dLN. Furthermore, our ongoing sequencing of pre-treatment lesions will enable monitoring of $\mathrm{T}$ cell clonal expansion, and additionally transcriptomic characterization of these samples will be correlated with post-treatment $\mathrm{T}$ cell programs to test predictive potential of baseline lymphoid phenotype. Correlations of these phenotypes with response to PD-1 blockade will allow for validation of predictive biomarkers, and characterizing these $\mathrm{T}$ cell programs in the dLN and the tumor microenvironment will enable superior and personalized therapeutic interventions.

Ethics Approval Samples of tumor and non-involved liver were obtained from surgical specimens of patients undergoing resection at Mount Sinai Hospital (New York, NY) after obtaining informed consent in accordance with a protocol reviewed and approved by the Institutional Review Board at the Icahn School of Medicine at Mount Sinai (RUTH Human Subjects Electronic Submission System 18-00407 and 20-04150) and in collaboration with the Biorepository and Department of Pathology.

http://dx.doi.org/10.1136/jitc-2021-SITC2021.361 